\title{
A UNIQUE OSCILLATION SOLUTION TO THE SOLAR NEUTRINO PROBLEM?
}

\author{
M. B. SMY \\ Department of Physics and Astronomy \\ 4182 Frederick Reines Hall, University of California, Irvine, CA 92697-4575
}

\begin{abstract}
A global two-neutrino oscillation fit combining Super-Kamiokande solar neutrino data with the solar neutrino rates measured by Homestake, Gallex/GNO, SAGE and SNO prefers a single allowed area, the Large Mixing Angle solution, at about $95 \%$ confidence level. The mass ${ }^{2}$ difference between the two mass eigenstates is $\Delta m^{2} \approx 3-25 \times 10^{-5} \mathrm{eV}^{2}$, the mixing angle $\theta$ is $\tan ^{2} \theta \approx 0.21-0.67$.
\end{abstract}

\section{Introduction}

Neutrino flavor oscillations are currently the most favored way to understand the larger solar ${ }^{8} \mathrm{~B}$ neutrino interaction rate measured by Super-Kamiokande $]$ (SK) with respect to the ${ }^{8} \mathrm{~B}$ rate reported by the SNO-collaboration $\mathrm{B}$. The SNO rate includes only e-type ${ }^{8} \mathrm{~B}$ neutrinos while SK measures solar neutrinos via neutrino-electron elastic scattering which has a small sensitivity to other (active) flavors as well. The SK excess is then interpreted as a hint for appearance of other active flavors in the ${ }^{8} \mathrm{~B}$ neutrino flux (born in the sun as purely $e$-type). Neutrino oscillations also explain the deficit of both rates as well as the deficits of the "Chlorine rate" (HomestakeB) and the "Gallium rates" (Gallex/GNOA, SAGEl) with respect to the Standard Solar Modelo (SSM). Chlorine and Gallium rates include only e-type solar neutrinos.

The large neutrino flavor mixing betyeen the second and third generation inferred from atmospheric neutrino datat and the absence of an oscillation signal in the CHOOZ reactor neutrino experiment $\mathrm{O}_{\text {requires the neutrino flavor }}$ mixing between the first and third generation to be small. Solar neutrino oscillations can therefore be approximated by a two-neutrino description with the parameters $\theta$ (mixing angle) and $\Delta m^{2}$ (difference in mass ${ }^{2}$ ). The mixing angle ranges between 0 and $\pi / 2$, since $\Delta m^{2}$ is defined to be positive. For $\Delta m^{2}$ between $\approx 10^{-8} \mathrm{eV}^{2}$ and $\approx 10^{-3} \mathrm{eV}^{2}$, the matter density in the sun and earth can strongly affect the oscillation probability. For $\theta<\pi / 4$, resonant enhancement of the oscillations (MSW effect 9 ) can occur in the sun. On the "dark side" of the parameter space $(\theta>\pi / 4)$, an anti-resonance can suppress the oscillations. Below $\approx 10^{-9} \mathrm{eV}^{2}$ (quasi-vacuum/vacuum region), the oscillation probability is more affected by the oscillation phase than by matter effects. 


\section{Parameter Estimation for Two-Neutrino Oscillations}

The simplest way to constrain the oscillation parameters is a $\chi^{2}$ fit to all $e$ type neutrino rates, that is Gallex/GNO and SAGE (combined into a single "Gallium" number), Homestake and SNO. The dotted lines of figure 1 a) show the allowed regions at 95\% C.L. (which can be understood as an "overlay" of the allowed regions 10 from the three experimental rates mentioned above and their SSM-based predictions). The area above $\Delta m^{2} \approx 10^{-5} \mathrm{eV}^{2}$ near maximal mixing is called the Large Mixing Angle (LMA) solution. The Small Mixing Angle Solution (SMA) is located between $\Delta m^{2} \approx 10^{-6} \mathrm{eV}^{2}$ and $\approx 10^{-5} \mathrm{eV}^{2}$ at $\tan ^{2} \theta \approx 10^{-3}$. The LOW solution is the large $\Delta m^{2}$ part of the extensive region(s) between $\approx 10^{-7} \mathrm{eV}^{2}$ and $\approx 10^{-9} \mathrm{eV}^{2}$. The lower part of this region is called the quasi-vacuum (quasi-VAC) solution. The vacuum solutions (VAC) are below $\approx 10^{-10} \mathrm{eV}^{2}$. All regions have similar $\chi^{2}$ (LOW fits slightly worse).
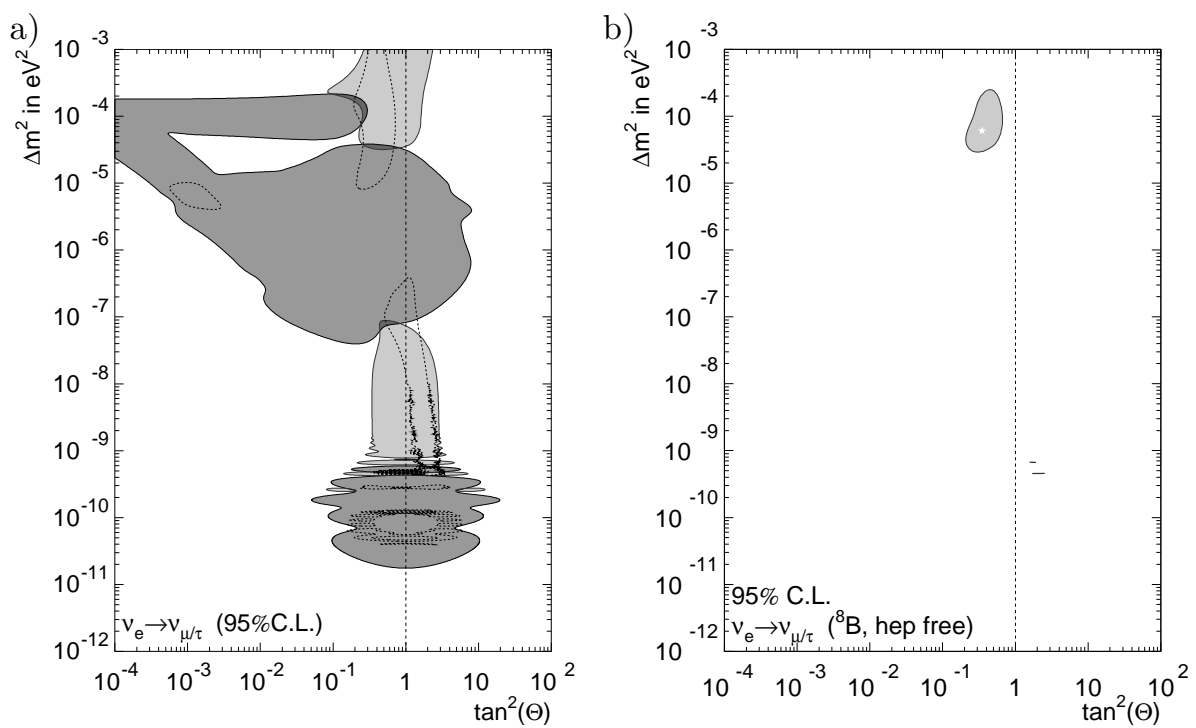

Figure 1. SK Zenith Spectrum/SNO (a) and global fit (b) contours at 95\% C.L. The dark-grey area in a) is excluded by the shape of the SK Zenith Spectrum. Adding the SNO rate measurement results in the (light-grey) allowed areas. Both regions do not depend on neutrino flux predictions by the SSM. Overlaid are the allowed regions (inside dotted lines) based on the Gallex/GNO, SAGE, Homestake and SNO rates and the SSM. In b) the allowed areas from a global fit (adding the rates of Gallex/GNO, SAGE and Homestake to the SK Zenith Spectrum-SNO fit) are predominantly LMA solutions. 
To break the degeneracy in $\chi^{2}$ of these regions, more information is needed than provided by the suppression of the $e$-type rates. Indeed, neutrino oscillations not only cause a 'disappearance' of the $e$-type fluxes but also produce other neutrino flavors (appearance). Furthermore, they can induce distortions of the neutrino spectrum and time variations of the solar neutrino fluxes. In the MSW region, the time variations arise from matter effects inside the earth (daily variations). In the vacuum regions, the time variations are the consequence of the change of the oscillation phase due to the yearly variation of the oscillation baseline (distance between sun and earth).

SK can search for spectral distortions through analysis of the spectrum of the recoiling electron as well as daily or yearly time variations. The analysis of the shape of the SK "zenith angle spectrum" 19.11 combines spectrum and daily variation analyses. Neither spectral distortion nor daily variation was found. The centerpiece of this work is the preliminary SK zenith angle spectrum based on the entire SK data set (1496 days). The numerical results are listed in tables 1 and 2. Figure 17a) shows the excluded regions (dark-grey) from the SK zenith angle spectrum (for an explanation of the fit method see Appendix, eq. 2). The SMA and VAC regions are excluded since they require a distorted ${ }^{8} \mathrm{~B}$ neutrino spectrum ${ }^{0}$. The lower part of the LMA and the upper part of the LOW solution predict daily variations 10 and are therefore disfavored. The excluded areas are independent of the SSM neutrino fluxes. The SK zenith angle spectrum shape is best descriped by quasi-VAC solutions.

If combined with the SNO rate 2 of $0.346_{-0.028}^{+0.029} \times \operatorname{SSM}$ (Appendix, eq. 3), the SK rate provides a probe for the appearance of other neutrino flavors. The SK rate of $0.465_{-0.013}^{+0.015} \times \mathrm{SSM}$ (see table 1) exceeds the $e$-type rate inferred from the SNO measurement by more than $3 \sigma$. If this is interpreted as appearance, the other flavors contribute about $25 \%$ to the SK rate and $70 \%$ to the ${ }^{8} \mathrm{~B}$ flux (the SK cross section for the other flavors is six to seven times smaller than for $e$-type neutrinos). The two light-grey allowed regions of figure (1a) are based on the combined fit to the SK zenith angle spectrum and the SNO rate 2 . One region contains the upper part of the LMA solution, the other region contains the lower part of the LOW solution and the quasi-VAC solutions (best-fit). The allowed areas are still independent of the SSM neutrino fluxes.

The Appendix (eq. (1) explains the method to add the Galliumb $\left(74.8_{-5.0}^{+5.1} \mathrm{SNU}\right.$ or $\left.0.584_{-0.039}^{+0.040} \times \mathrm{SSM}\right)$ and Chlorine rates $3(2.56 \pm 0.23 \mathrm{SNU}$ or $0.337 \pm 0.030 \times \mathrm{SSM}$ ) to the fit. The allowed areas of this global fit (shown in figure $\mathrm{I} \mathrm{b}$ ) look quite different when compared with the $e$-type rate fit: Only the upper part of the LMA survives (and two tiny quasi-VAC solutions). The disappearance of the LOW solution is due to predicted daily variations. The SK rate requires a larger ${ }^{8} \mathrm{~B}$ flux than the Chlorine rate allows, so almost all 
Table 1. SK Rate and Uncertainty for eight energy bins. The rates, statistical and systematic uncertainties (of the spectrum shape) in the third column are presented in units of SSM expectation. These systematic uncertainties are assumed to be uncorrelated in energy. The uncertainties in the fourth (uncertainty of the ${ }^{8} \mathrm{~B}$ neutrino spectrum), fifth (uncertainty of the energy scale of $0.64 \%$ ) and sixth column (uncertainty of the energy resolution of $2.5 \%$ ) are fully correlated in energy (but uncorrelated with each other). The combined uncertainty (last row) is based on the sum of all bins of the zenith angle spectrum. The combined rate has an additional systematic uncertainty of $\sigma_{S K}={ }_{-2.6}^{+2.9} \%$ (excluding ${ }^{8} \mathrm{~B}$ shape, energy scale and resolution), which was added to the uncorrelated systematic uncertainties of the spectrum shape.

\begin{tabular}{|c|c|c|c|c|c|}
\hline Bin & Range $[\mathrm{MeV}]$ & Rate \pm stat \pm syst $[\mathrm{SSM}]$ & ${ }^{8} \mathrm{~B}$ Spectrum & E-Scale & E-Resol. \\
\hline 1 & $5.0-5.5$ & $0.4671 \pm 0.0404_{-0.0138}^{+0.0165}$ & $\begin{array}{l}+0.04 \% \\
-0.02\end{array}$ & $\begin{array}{l}+0.09 \% \\
-0.01 \%\end{array}$ & $\begin{array}{l}+0.23 \% \\
-0.21\end{array}$ \\
\hline 2 & $5.5-6.5$ & $0.4580 \pm 0.0141_{-0.0065}^{+0.0066}$ & $\begin{array}{l}+0.13 \% \\
-0.09 \%\end{array}$ & $\begin{array}{l}+0.20 \% \\
-0.16 \%\end{array}$ & $\begin{array}{l}+0.19 \% \\
-0.17\end{array}$ \\
\hline 3 & $6.5-8.0$ & $0.4729 \pm 0.0084 \pm 0.0065$ & $\begin{array}{l}+0.41 \% \\
-0.38 \%\end{array}$ & $\begin{array}{l}+0.63 \% \\
-0.62\end{array}$ & $\begin{array}{l}+0.17 \% \\
-0.16\end{array}$ \\
\hline 4 & $8.0-9.5$ & $0.4599 \pm 0.0093 \pm 0.0063$ & $\begin{array}{l}+0.89 \\
-0.85 \%\end{array}$ & $\begin{array}{l}+1.3 \% \\
-1.3\end{array}$ & $\begin{array}{l}+0.12 \\
-0.12\end{array}$ \\
\hline 5 & $9.5-11.5$ & $0.4627 \pm 0.0103 \pm 0.0063$ & $+1.7 \%$ & $+2.5 \%$ & $\begin{array}{l}+0.16 \\
-0.18\end{array}$ \\
\hline 6 & $11.5-13.5$ & $0.4621 \pm 0.0168 \pm 0.0063$ & $\begin{array}{l}+3.1 \% \\
-2.7\end{array}$ & $+4.4 \%$ & ${ }_{-1.1}^{+1.1} \%$ \\
\hline 7 & $13.5-16.0$ & $0.5666 \pm 0.0390 \pm 0.0078$ & $+5.1 \%$ & $\begin{array}{l}+7.0 \\
-6.4\end{array}$ & $+3.2 \%$ \\
\hline 8 & $16.0-20.0$ & $0.5554 \pm 0.1458 \pm 0.0076$ & $\begin{array}{l}+7.7 \% \\
-5.6\end{array}$ & $\begin{array}{l}+10.6 \% \\
-\quad 9.6 \%\end{array}$ & $\begin{array}{l}+8.4 \\
-7.9\end{array}$ \\
\hline \multicolumn{2}{|c|}{ Comb. 5.0-20.0 } & $0.4653 \pm 0.0047_{-0.0122}^{+0.0138}$ & $\begin{array}{l}+1.15 \% \\
-1.04 \%\end{array}$ & $\begin{array}{l}+1.66 \% \\
-1.58 \%\end{array}$ & $\begin{array}{l}+0.33 \% \\
-0.34\end{array}$ \\
\hline
\end{tabular}

Table 2. Subdivision of bins 2-7 according to the solar zenith angle $\theta_{z}$. The range of $\cos \theta_{z}$ is given for each bin: $\cos \theta_{z}<0$ is 'Day' and $\cos \theta_{z}>0$ is 'Night' ('Mantle' and 'Core'). The rates are given in units of $0.001 \times \mathrm{SSM}$. Only statistical uncertainties are quoted. All systematic uncertainties (see table 1 ) are assumed to be fully correlated in zenith angle.

\begin{tabular}{|c|c|c|c|c|c|c|c|}
\hline Bin & $\begin{array}{l}\text { Day } \\
-1-0\end{array}$ & $0.00-0.16$ & $0.16-0.33$ & $\begin{array}{c}\text { Mantle } \\
0.33-0.50\end{array}$ & $0.50-0.67$ & $0.67-0.84$ & $\begin{array}{c}\text { Core } \\
0.84-1\end{array}$ \\
\hline 2 & $453 \pm 20$ & $442 \pm 53$ & $379 \pm 49$ & $472 \pm 45$ & $522 \pm 45$ & $503 \pm 49$ & $426 \pm 52$ \\
\hline 3 & $474 \pm 12$ & $530 \pm 34$ & $506 \pm 30$ & $438 \pm 26$ & $478 \pm 26$ & $451 \pm 28$ & $439 \pm 31$ \\
\hline 4 & $448 \pm 13$ & $463 \pm 36$ & $470 \pm 33$ & $462 \pm 29$ & $509 \pm 29$ & $461 \pm 32$ & $451 \pm 35$ \\
\hline 5 & $453 \pm 15$ & $449 \pm 40$ & $502 \pm 38$ & $451 \pm 32$ & $473 \pm 32$ & $477 \pm 35$ & $483 \pm 40$ \\
\hline 6 & $477 \pm 25$ & $509 \pm 67$ & $351 \pm 55$ & $391 \pm 49$ & $498 \pm 53$ & $434 \pm 56$ & $521 \pm 64$ \\
\hline 7 & $511 \pm 54$ & $570 \pm 150$ & $831 \pm 167$ & $694 \pm 131$ & $665 \pm 127$ & $441 \pm 118$ & $469 \pm 131$ \\
\hline
\end{tabular}

the quasi-VAC regions disappear. The fit does not depend on the ${ }^{8} \mathrm{~B}$ and hep neutrino flux predictions by the SSM, which suffer from the largest uncertainties. However, it depends on the other SSM neutrino fluxes, in particular the ${ }^{7}$ Be flux (10\% uncertainty) and the neutrino fluxes of the CNO cycle $(\approx 20 \%$ uncertainty). Those fluxes contribute $\mathrm{\theta}$ about $15 \%\left({ }^{7} \mathrm{Be}\right)$ and $6 \%(\mathrm{CNO})$ to the Chlorine rate and $27 \%\left({ }^{7} \mathrm{Be}\right)$ and $7 \%(\mathrm{CNO})$ to the Gallium rate in the SSM. 
Table 3. Parameters for the best-fit points. The probabilities given in the fourth row are based on the difference of $\chi^{2}$ (with respect to the minimum). The five rows below show the five independent parts of the fit: the $\Delta \chi^{2}$ from a fit to the shape of the SK zenith spectrum (Appendix, eq. 2) and four interaction rates (deviation probabilities are given in units of Gaussian standard deviation $\sigma$ ). The last four rows show the values of the minimized fit parameters. The ${ }^{8} \mathrm{~B}$ and the hep fluxes are free, the ${ }^{8} \mathrm{~B}$ neutrino spectrum shift as well as the SK energy scale and resolution shifts are constrained within the systematic uncertainties.

\begin{tabular}{|l|cccc|}
\hline Solution & $\begin{array}{c}\text { Large Mixing } \\
\text { Angle }(\mathrm{LMA})\end{array}$ & $\begin{array}{c}\text { Quasi-Vacuum } \\
(\text { Quasi-VAC) }\end{array}$ & $\begin{array}{c}\text { Low } \Delta m^{2} \\
(\mathrm{LOW})\end{array}$ & $\begin{array}{c}\text { Small Mixing } \\
\text { Angle }(\mathrm{SMA})\end{array}$ \\
\hline$\Delta m^{2}$ & $6.0 \times 10^{-5}$ & $4.57 \times 10^{-10}$ & $5.0 \times 10^{-8}$ & $4.8 \times 10^{-6}$ \\
$\tan ^{2} \theta$ & 0.35 & 2.1 & 0.83 & 0.00044 \\
\hline$\chi^{2}\left(45\right.$ dof; $\left.p_{\chi^{2}}[\%]\right)$ & $43.4(53.9)$ & $48.5(33.4)$ & $51.2(24.3)$ & $54.2(16.2)$ \\
$\Delta \chi^{2}\left(2\right.$ dof; $\left.p_{\Delta \chi^{2}}[\%]\right)$ & $0.0(100.0)$ & $5.1(7.9)$ & $7.8(2.0)$ & $10.8(0.5)$ \\
\hline$\Delta \chi_{S K}^{2}\left(p_{\Delta \chi^{2}}[\sigma]\right)$ & $3.4(1.3 \sigma)$ & $3.1(1.3 \sigma)$ & $3.9(1.5 \sigma)$ & $5.0(1.7 \sigma)$ \\
Ga Rate $[\mathrm{SNU}]$ & $73.2(-0.3 \sigma)$ & $69.6(-1.0 \sigma)$ & $68.2(-1.3 \sigma)$ & $75.1(+0.1 \sigma)$ \\
Cl Rate $[\mathrm{SNU}]$ & $2.97(+1.8 \sigma)$ & $3.18(+2.8 \sigma)$ & $3.13(+2.5 \sigma)$ & $2.67(+0.5 \sigma)$ \\
SK Rate $[\% \mathrm{SSM}]$ & $46.4(-0.1 \sigma)$ & $44.7(-1.4 \sigma)$ & $44.9(-1.2 \sigma)$ & $44.1(-1.9 \sigma)$ \\
SNO Rate $[\% \mathrm{SSM}]$ & $32.8(-0.7 \sigma)$ & $37.1(+0.8 \sigma)$ & $38.5(+1.3 \sigma)$ & $43.8(+3.1 \sigma)$ \\
\hline$\phi_{8}\left[10^{6} /\left(\mathrm{cm}^{2} \mathrm{~s}\right)\right]$ & $5.62(+0.6 \sigma)$ & $3.71(-1.7 \sigma)$ & $4.04(-1.2 \sigma)$ & $2.71(-2.9 \sigma)$ \\
$\phi_{h e p}\left[10^{3} /\left(\mathrm{cm}^{2} \mathrm{~s}\right)\right]$ & 40 & 0 & 21 & 8 \\
${ }^{8} \mathrm{~B}$ Spectrum Shape & $-0.3 \sigma$ & $-0.7 \sigma$ & $-0.1 \sigma$ & $+0.1 \sigma$ \\
SK E-scale/resol. & $-0.4 \sigma /-0.1 \sigma$ & $-1.0 \sigma / 0.0 \sigma$ & $-0.1 \sigma /-0.2 \sigma$ & $+0.1 \sigma /-0.4 \sigma$ \\
\hline
\end{tabular}

\section{Results of the global fit}

Table 3 compares the four smallest local minima of the $\chi^{2}$ describing the fit. The best-fit is located in the upper LMA area. The ${ }^{8} \mathrm{~B}$ flux resulting from this fit is somewhat higher than expected by the SSM $\left(5.05_{-0.81}^{+1.01} \times 10^{6} /\left(\mathrm{cm}^{2} \mathrm{~s}\right)\right)$ but well within the uncertainty. The hep flux is considerably higher than expected by the $\operatorname{SSM}\left(9.3 \times 10^{3} /\left(\mathrm{cm}^{2} \mathrm{~s}\right)\right)$; however, the uncertainty of this prediction is thought to be very large. The fit agrees with the SK zenith angle spectrum moderately well. Figure 2a) shows the SK zenith angle spectrum and this best fit. Figure $2 \mathrm{~b}$ ) gives a magnified view of the LMA region (in a linear scale of $\tan ^{2} \theta$ ). The $1 \sigma$ (black) and $3 \sigma$ (light grey) contours are also shown. The fit accommodates well the Gallium, SK and SNO rates, however the predicted Chlorine rate is about $2 \sigma$ too high. This worsens the otherwise very good best-fit $\chi^{2}$. As a consequence, quasi-VAC solutions, which don't fit well the interaction rates in general, appear at 95\% C.L.

The quasi-VAC solution describes the SK zenith angle spectrum slightly better than the LMA. The fit is helped by a statistical up-fluctuation of the last two bins (see figure 3). Together with a down-shift of energy scale and ${ }^{8} \mathrm{~B}$ spectrum, the required oscillation minimum can be 'generated' in the data. 

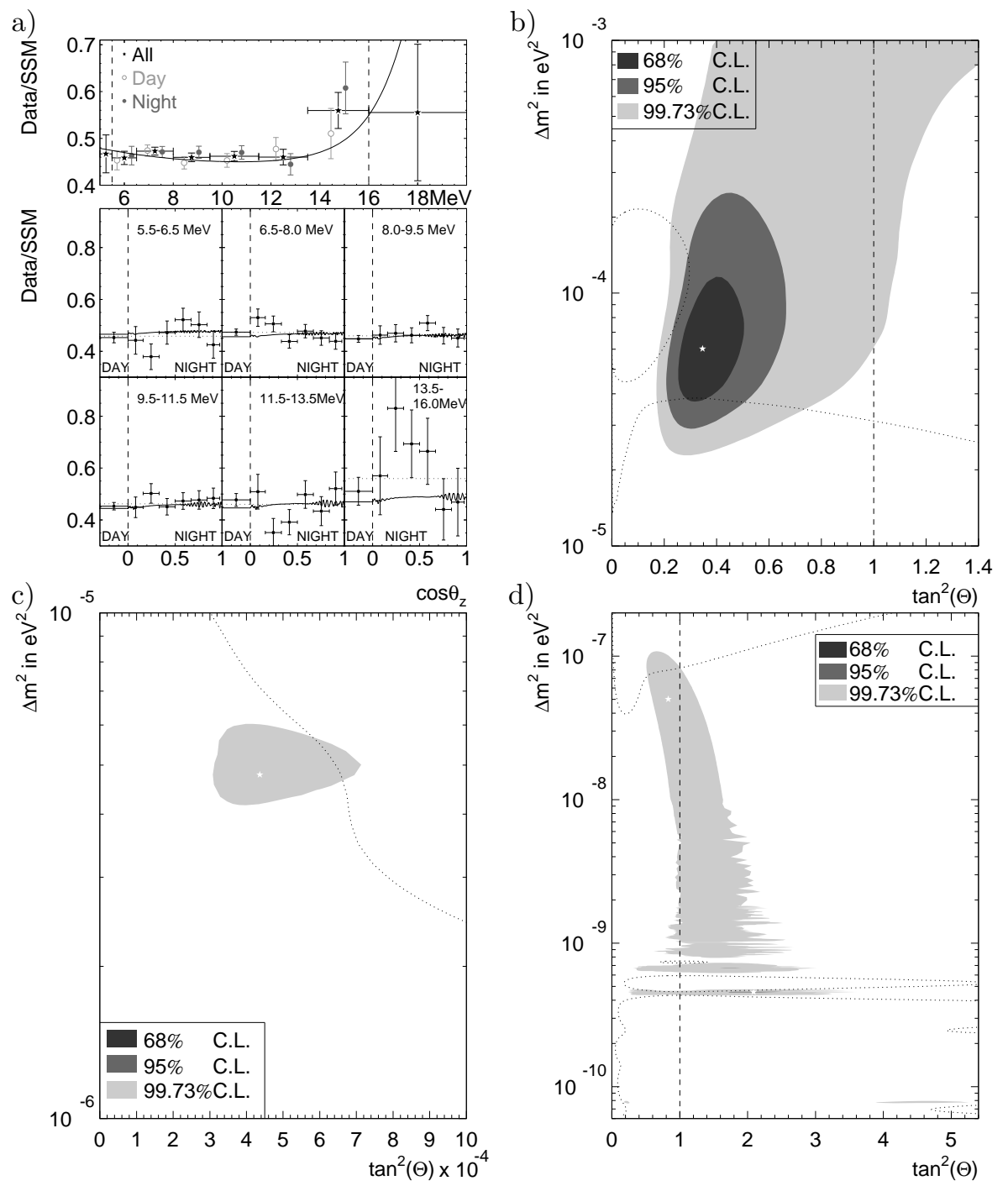

Figure 2. SK Zenith Spectrum (a) and LMA (b), SMA (c) and LOW/Quasi-VAC (d) contours resulting from the global fit. The top panel of a) shows the SK energy spectrum during the day (open circles), the night (filled circles) as well as day and night combined (stars). Between 5.5 and $16 \mathrm{MeV}$, zenith-angle distributions are shown (lower 6 panels of a). The dotted line in each of the lower panels is the combined rate in that bin. Error bars reflect the statistical uncertainty only. Superimposed is an oscillation prediction $\left(\tan ^{2} \theta=0.34\right.$ and $\Delta m^{2}=6 \cdot 0 \cdot 10^{-5} \mathrm{eV}^{2}$ ) near the best-fit point. In b)-d) the one (black), two (dark-grey) and three $\sigma$ (light-grey) contours of the LMA, SMA and LOW/Quasi-VAC solutions are shown in a linear scale in $\tan ^{2} \theta$. The best fits are indicated by the white stars. Superimposed is the 95\%C.L. excluded area from the SK Zenith Spectrum (inside dotted lines). SMA, LOW and Quasi-VAC are disfavored. 
The rates, however, do not fit well. The fit struggles to accommodate both the Chlorine and SK rate. In this region Chlorine and SK disagree by $3 \sigma$ about the ${ }^{8} \mathrm{~B}$ flux (which in the un-oscillated SSM contributes about $76 \%$ of the Chlorine rate). The resulting best-fit ${ }^{8} \mathrm{~B}$ flux falls $1.7 \sigma$ short of the SSM prediction. Even though the quasi-VAC solution has an overall 'C.L. threshold' of $8 \%$, it is considerably disfavored, when thus checked in detail. The surviving (at 98\% C.L.) LOW solution fits the rates about as poorly as the quasi-VAC solution. The SK zenith angle spectrum fits somewhat worse than either LMA or quasi-VAC. The lack of zenith angle variation in the SK data reduces the $\Delta m^{2}$ (usually around $10^{-7} \mathrm{eV}^{2}$ ) and worsens the LOW best-fit which is already under pressure from the rates. Figure $2 \mathrm{~d}$ ) shows an enlarged view of the LOW and quasi-VAC region.

A "smaller-than-small mixing angle" solution appears just left of the SMA at about the $3 \sigma$ level (see figure 2 $\mathrm{c}$ ). The SMA region is defined by the crossing of the Gallium and the Chlorine allowed area (for a given ${ }^{8} \mathrm{~B}$ flux). It therefore fits those two rates very well. The SK zenith angle spectrum, however, fits the worst of all solutions: The SK spectral data lack the predicted distortion. The SNO rate is more than $3 \sigma$ above the measurement, the SK rate is too low $(2 \sigma)$. The ${ }^{8} \mathrm{~B}$ flux required is very low $(3 \sigma)$.

The ${ }^{8} \mathrm{~B}$ flux comparisons above refer to the SSM value. A recent precision measurement of the cross section of the ${ }^{7} \mathrm{Be}(\mathrm{p}, \gamma)^{8} \mathrm{~B}$ fusion reaction by Junghans et al.13 implies (see 4 ) a flux of $5.93_{-0.89}^{+0.83} \times 10^{6} /\left(\mathrm{cm}^{2} \mathrm{~s}\right.$ ). If the fit is confined to this flux, then all solutions other than LMA are further disfavored.

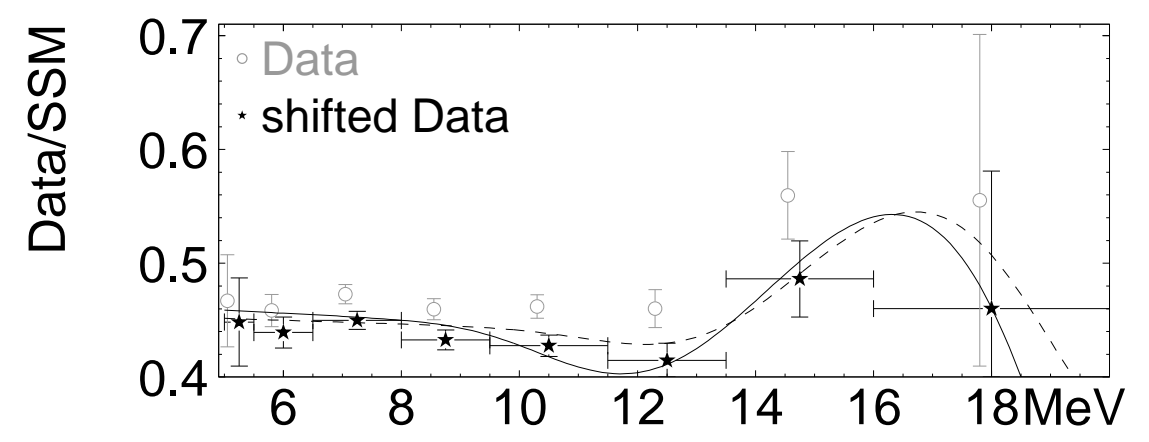

Figure 3. SK Spectral distortion for two quasi-VAC solutions (solid line: best fit at 4.57 . $10^{-10} \mathrm{eV}^{2}$, dashed line: $6.68 \cdot 10^{-10} \mathrm{eV}^{2}$ ) and data (open circles). Although the data do not support spectral distortions, a shift (stars) in energy scale $(-1.0 \sigma)$ and ${ }^{8} \mathrm{~B}$ shape $(-0.7 \sigma)$ and systematic uncertainty of the combined rate $(-1.4 \sigma)$ can accommodate the prediction. 


\section{Conclusion}

A global fit to all solar neutrino data was performed using a two-neutrino oscillation model. At 95\% C.L. only the upper LMA solution and two tiny quasi-VAC regions are still allowed. The quasi-VAC regions are disfavored at more than $92 \%$ C.L. caused by a disagreement about the ${ }^{8} \mathrm{~B}$ flux between SK and the Chlorine rate of $3 \sigma$; the resulting quasi-VAC ${ }^{8} \mathrm{~B}$ flux fit is $1.7 \sigma$ smaller than the Standard Solar Model. The LMA solutions above $\Delta m^{2}=3 \cdot 10^{-5} \mathrm{eV}^{2}$ are therefore the only viable solution at $95 \%$ C.L.

\section{Acknowledgments}

This analysis relies crucially on Super-Kamiokande data. The author acknowledges the extensive help and cooperation of the Super-Kamiokande collaboration as well as the Kamioka Mining and Smelting Company. The Super-Kamiokande detector has been built and operated from funding by the Japanese Ministry of Education, Culture, Sports, Science and Technology, the U.S. Department of Energy, and the U.S. National Science Foundation.

\section{Appendix: Fitting Method}

To estimate and limit oscillation parameters, a $\chi^{2}$ describing the shape of the SK zenith spectrum is extended to take into account the measured neutrino interaction rates by various experiments. Using oscillation probabilities (obtained as in 11) and SSM neutrino fluxes, the expected interaction rates $B_{i, z}^{\text {osc }}$ (due to ${ }^{8} \mathrm{~B}$ neutrinos) and $H_{i, z}^{\text {osc }}$ (due to hep neutrinos) in energy bin $i$ and zenith-angle bin $z$ are calculated. These rates as well as the SK measurements $D_{i, z}$ are then normalized by the SSM expectations without oscillations:

$$
b_{i, z}=\frac{B_{i, z}^{\mathrm{osc}}}{B_{i, z}^{\mathrm{SSM}}+H_{i, z}^{\mathrm{SSM}}}, h_{i, z}=\frac{H_{i, z}^{\mathrm{osc}}}{B_{i, z}^{\mathrm{SSM}}+H_{i, z}^{\mathrm{SSM}}}, d_{i, z}=\frac{D_{i, z}}{B_{i, z}^{\mathrm{SSM}}+H_{i, z}^{\mathrm{SSM}}} .
$$

The zenith components of the flux difference vector $\overrightarrow{\Delta_{i}}$

$$
\Delta_{i, z}(\beta, \eta)=\left(\beta \cdot b_{i, z}+\eta \cdot h_{i, z}\right) \times f\left(E_{i}, \delta_{B}, \delta_{S}, \delta_{R}\right)-d_{i, z}
$$

allow for arbitrary neutrino fluxes (through the free parameters $\beta$ and $\eta$ ). The combined rate prediction is modified by the energy-shape factors

$$
f\left(E_{i}, \delta_{B}, \delta_{S}, \delta_{R}\right)=f_{B}\left(E_{i}, \delta_{B}\right) \times f_{S}\left(E_{i}, \delta_{S}\right) \times f_{R}\left(E_{i}, \delta_{R}\right)
$$

with $\delta_{B}$ describing the ${ }^{8} \mathrm{~B}$ neutrino spectrum shape uncertainty, $\delta_{S}$ describing the uncertainty of the SK energy scale (0.64\%) and $\delta_{R}$ describing the 
uncertainty of the SK energy resolution $(2.5 \%)$ (The shapes $f_{B}, f_{S}, f_{R}$ are given in table 1). All three uncertainties affect the bins of the SK zenith angle spectrum in a correlated way. The $7 \times 7$ matrices $V_{i}$ describe statistical and energy-uncorrelated uncertainties; the latter are assumed to be fully correlated in zenith angle. For any given parameters $\delta_{k}$, the $\chi^{2}$

$$
\begin{gathered}
\chi_{0}^{2}=\sum_{i=1}^{8} \overrightarrow{\Delta_{i}} \cdot V_{i}^{-1} \cdot \vec{\Delta}_{i}=\chi_{0, m}^{2}+\left(\vec{\phi}-\vec{\phi}_{0, m}\right) C_{0}\left(\vec{\phi}-\vec{\phi}_{0, m}\right) \\
\text { with } \quad C_{0}=\sum_{i=1}^{8}\left(\begin{array}{ll}
\overrightarrow{b_{i}} \cdot V_{i}^{-1} \cdot \overrightarrow{b_{i}} & \overrightarrow{h_{i}} \cdot V_{i}^{-1} \cdot \overrightarrow{b_{i}} \\
\overrightarrow{h_{i}} \cdot V_{i}^{-1} \cdot \overrightarrow{b_{i}} & \overrightarrow{h_{i}} \cdot V_{i}^{-1} \cdot \overrightarrow{h_{i}}
\end{array}\right) \quad \text { and } \quad \vec{\phi}=\left(\begin{array}{c}
\beta \\
\eta
\end{array}\right)
\end{gathered}
$$

can be written as a quadratic form of $\vec{\phi}$ and the curvature matrix $C_{0}$. The minimum is

$$
\chi_{0, m}^{2}=\sum_{i=1}^{8} \overrightarrow{d_{i}} \cdot V_{i}^{-1} \cdot \vec{d}_{i}-C_{0, m} \quad \text { with } \quad C_{0, m}=\vec{\phi}_{0, m} C_{0} \vec{\phi}_{0, m} .
$$

If the minimum flux vector is scaled by $\alpha\left(\vec{\phi}=\alpha \times \vec{\phi}_{0, m}\right)$ then $\chi_{0}^{2}$ constrains $\alpha$ to be $\alpha=1 \pm \sqrt{1 / C_{0, m}}$. To take into account the systematic uncertainty of the SK combined rate $\sigma_{S K}={ }_{-2.6}^{+2.9} \%$ (which is completely correlated in zenith-angle and energy), $\chi_{0}^{2}$ is modified to

$\chi_{1}^{2}=\chi_{0, m}^{2}+\left(\vec{\phi}-\vec{\phi}_{0, m}\right) C_{1}\left(\vec{\phi}-\vec{\phi}_{0, m}\right) \quad$ with $\quad C_{1}=\frac{1 / \sigma_{S K}^{2}}{C_{0, m}+1 / \sigma_{S K}^{2}} \times C_{0}$.

$\chi_{1}^{2}$ constrains $\alpha$ to be $\alpha=1 \pm \sqrt{1 / C_{0, m}+\sigma_{S K}^{2}}$, that is, the minimum is unchanged, but the allowed parameter range for $\beta$ and $\eta$ is larger. The total $\chi^{2}$ for the SK zenith spectrum shape is then

$$
\chi_{\mathrm{SK}}^{2}=\operatorname{Min}\left(\chi_{1}^{2}\left(\beta, \eta, \delta_{B}, \delta_{S}, \delta_{R}\right)+\left(\frac{\delta_{B}}{\sigma_{B}}\right)^{2}+\left(\frac{\delta_{S}}{\sigma_{S}}\right)^{2}+\left(\frac{\delta_{R}}{\sigma_{R}}\right)^{2}\right)
$$

where all $\delta_{k}$ as well as $\beta, \eta$ are minimized.

A combined fit with the rate measured by the SNO collaboration 3 can be done by defining $b_{\mathrm{SNO}}, h_{\mathrm{SNO}}$ and $\Delta_{\mathrm{SNO}}(\beta, \eta)$ in a similar way as in (11) and form

$$
\chi_{\mathrm{SK}-\mathrm{SNO}}^{2}=\operatorname{Min}\left(\chi_{1}^{2}+\left(\frac{\delta_{B}}{\sigma_{B}}\right)^{2}+\left(\frac{\delta_{S}}{\sigma_{S}}\right)^{2}+\left(\frac{\delta_{R}}{\sigma_{R}}\right)^{2}+\left(\frac{\Delta_{\mathrm{SNO}}(\beta, \eta)}{\sigma_{\mathrm{SNO}}}\right)^{2}\right)
$$


To add the radio-chemical rate measurements of Homestake 3 , Gallex/GNOl, and SAGEE (all "Gallium" rates are combined into a single rate), the $\chi^{2}$

$$
\chi_{\mathrm{RC}}^{2}(\beta, \eta)
$$

takes into account the correlations between Gallium and Chlorine measurements. The ${ }^{8} \mathrm{~B}$ and hep fluxes are constrained by the minimization of $\chi_{\mathrm{SK}-\mathrm{SNO}}^{2}$, not by the Standard Solar Model. The total $\chi^{2}$ is then a simple addition

$$
\chi^{2}=\chi_{\mathrm{SK}-\mathrm{SNO}}^{2}+\operatorname{Min}\left(\chi_{\mathrm{RC}}^{2}\left(\alpha \beta_{\min }, \alpha \eta_{\min }\right)+\left(\frac{\alpha-1}{\sigma_{\alpha}}\right)^{2}\right)
$$

where $1 / \sigma_{\alpha}^{2}=C_{\mathrm{SK}-\mathrm{SNO}, \mathrm{m}}=\vec{\phi}_{\mathrm{SK}-\mathrm{SNO}, \mathrm{m}} \cdot C_{\mathrm{SK}-\mathrm{SNO}} \cdot \vec{\phi}_{\mathrm{SK}-\mathrm{SNO}, \mathrm{m}}$. Numerically, $\sigma_{\alpha}$ is found to be about $2.7 \%$ (The accuracy of SK and SNO combined)

\section{References}

1. S. Fukuda et al., Phys. Rev. Lett. 86, 5651 (2001).

2. Q.R. Ahmad et al. Phys. Rev. Lett. 87, 71301 (2001).

3. B.T. Cleveland et al., Astrophys. J. 496, 505 (1998).

4. E. Bellotti, Nucl. Phys. B(Proc. Suppl.) 91, 44 (2001);

W. Hampel et al., Phys. Lett. B 388, 364 (1996);

P. Anselmann et al., Phys. Lett. B 342, 440 (1995).

5. V. Gavrin, Nucl. Phys. B(Proc. Suppl.) 91, 36 (2001);

J.N. Abdurashitov et al., Phys. Lett. B 328, 234 (1994).

6. J.N. Bahcall, M.H. Pinsonneault, S. Basu, Astrophys. J. 555, 990 (2001).

7. Y. Fukuda et al., Phys. Rev. Lett. 81, 1562 (1998).

8. M. Apollonio, Phys. Lett. B 466, 415 (1999).

9. S.P. Mikheyev and A.Y. Smirnov, Sov. Jour. Nucl. Phys. 42, 913 (1985); L. Wolfenstein, Phys. Rev. D 17, 2369 (1978).

10. For individual allowed areas, predictions of the spectrum and daily variations of oscillation solutions as well as a discussion of zenith angle spectrum vs. day/night spectrum or spectrum see M. Smy, hep-ex/0106064, to be published in proceedings of XXXVIth Rencontres de Moriond on Electroweak Interactions and Unified Theories.

11. S. Fukuda et al., Phys. Rev. Lett. 86, 5656 (2001).

12. M. Smy in Neutrino Oscillations in Venice, ed. M. Baldo Ceolin, (Venezia, 2001) 35.

13. A.R. Junghans et al., Phys. Rev. Lett. 88, 041101 (2002).

14. J.N. Bahcall, M.C. Gonzales-Garcia and Carlos Peña Garay, hepph/0111150 (2001). 